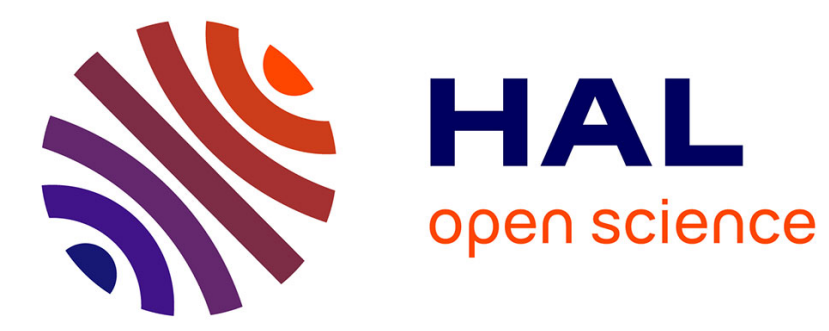

\title{
Multi-Output Dependent Observability Normal Form
}

Gang Zheng, Driss Boutat, Jean-Pierre Barbot

\section{To cite this version:}

Gang Zheng, Driss Boutat, Jean-Pierre Barbot. Multi-Output Dependent Observability Normal Form. Nonlinear Analysis: Theory, Methods and Applications, 2009, 70 (1), pp.404-418. 10.1016/j.na.2007.12.012 . inria-00192960v2

\section{HAL Id: inria-00192960 https://hal.inria.fr/inria-00192960v2}

Submitted on 2 Dec 2007

HAL is a multi-disciplinary open access archive for the deposit and dissemination of scientific research documents, whether they are published or not. The documents may come from teaching and research institutions in France or abroad, or from public or private research centers.
L'archive ouverte pluridisciplinaire HAL, est destinée au dépôt et à la diffusion de documents scientifiques de niveau recherche, publiés ou non, émanant des établissements d'enseignement et de recherche français ou étrangers, des laboratoires publics ou privés. 


\title{
Multi-Output Dependent Observability Normal Form
}

\author{
G. ZHENG $^{1}$, D. BOUTAT ${ }^{2}$ and J.P. BARBOT ${ }^{3}$ \\ ${ }^{1}$ INRIA Rhone-Alpes, Inovallée, 655 avenue de l'Europe, Montbonnot Saint \\ Martin, 38334 St. Ismier Cedex, France. \\ ${ }^{2}$ LVR/ENSI, 10 Boulevard de Lahitolle, 18020 Bourges, France. \\ ${ }^{3}$ ECS/ENSEA, 6 Avenue du Ponceau, 95014 Cergy-Pontoise, Project ALIEN, \\ INRIA-Futures, France.
}

\begin{abstract}
This article gives the sufficient condition which guarantees the existence of a reference frame in which a multi-output nonlinear system is linearizable with a linear part depending on its outputs. Our method is based on the design of a reference frame associated with nonlinear observable systems. Moreover, we give the generalization of the result obtained in [5] and [19]. And some examples are given in order to highlight our thinking.
\end{abstract}

Key words: normal form, observer design, linearization, left invertibility

\section{Introduction}

Given a nonlinear system, it is clear that no general approach can be used to design an observer. However, at least there are two ways to try. The first way is to design an observer directly to the given nonlinear system, including Kalman-like observer [8], adaptive observer [3], sliding mode observer [13] and so on. Nevertheless, some extra conditions should be imposed for these direct approaches, such as Lipschitz, persistent excitation. The second method is based on the conception of normal form. A normal form represents a class of equivalent systems which possess the same properties (we focus on the observability in this paper). In other words, the appearance differences among this class of equivalent systems are not intrinsic, but because of the wrong choice of the coordinates.

Obviously one of the keys of this category is to find out a coordinate transformation with which a given system could be converted into the target observ- 
able normal form. And then the existed nonlinear observer design techniques can be easily applied. In this category, $[3,1,2]$ deal with the problem of transform a nonlinear system into a class of state affine 'normal forms'. In fact, in their works, the sense of normal form is more general since no structure constraints were imposed for its linear part. However, it is also because of this merit, some existed techniques for linear systems cannot be easily applied. And this leads to the raise of linearization problem of nonlinear system.

The linearization problem with output injection was firstly treated in [10] for single output system. And it was generated in multi-output systems in $[11,17]$. Some other results about input-output injection for the linearization problem were stated in $[6,14]$. Moreover, $[15,16]$ gave the sufficient and necessary geometrical conditions to transform a nonlinear system into the so-called output-dependent time scaling linear canonical form. In [7], the author gave independently the dual geometrical conditions of [15]. Furthermore, the geometrical conditions to guarantee the existence of a local diffeomorphism and an output injection to transform a nonlinear system in a 'canonical' normal form depending on its output was presented [18][19], called Single Output Dependent Observability normal form (SODO). Moreover, an extension for this normal form with quadratic terms was also studied in [20]. As another natural extension for our previous study, we will extend our result for multi-outputs nonlinear system.

Motivated by this interest, this paper focuses on the analysis of nonlinear systems with multiple outputs as follows:

$$
\left\{\begin{array}{l}
\dot{x}=f(x, u) \\
y=\left(h_{1}(x), \ldots, h_{m}(x)\right)^{T}
\end{array}\right.
$$

where $x \in \mathbb{R}^{n}, u \in \mathbb{R}^{p}, f: \mathbb{R}^{n} \times \mathbb{R}^{p} \rightarrow \mathbb{R}^{n}$ and $h: \mathbb{R}^{n} \rightarrow \mathbb{R}^{m}$ are analytic, and we deal with the following problem: Find sufficient condition for the existence of a local diffeomorphism $\phi(x)=z$ to transform system (1) into

$$
\left\{\begin{array}{l}
\dot{z}=A(y, u) z+\beta(y, u) \\
y=C z
\end{array}\right.
$$


where

$$
z=\left[\begin{array}{c}
z_{1} \\
z_{2} \\
\vdots \\
z_{m}
\end{array}\right], \beta(y)=\left[\begin{array}{c}
\beta_{1}\left(y_{1}, u\right) \\
\beta_{2}\left(y_{1}, y_{2}, u\right) \\
\vdots \\
\beta_{m}\left(y_{1}, \ldots, y_{m}, u\right)
\end{array}\right], C=\left[\begin{array}{c}
C_{1} \\
C_{2} \\
\vdots \\
C_{m}
\end{array}\right]
$$

and

$$
A(y, u)=\left[\begin{array}{cccc}
A_{1}\left(y_{1}, u\right) & 0 & \cdots & 0 \\
0 & A_{2}\left(y_{1}, y_{2}, u\right) & \cdots & 0 \\
\vdots & \vdots & \ddots & \vdots \\
0 & 0 & \cdots & A_{m}\left(y_{1}, \ldots, y_{m}, u\right)
\end{array}\right]
$$

with

$$
z_{i}=\left[\begin{array}{c}
z_{i, 1} \\
z_{i, 2} \\
\vdots \\
z_{i, k_{i}}
\end{array}\right], \beta_{i}\left(y_{1}, \ldots, y_{i}, u\right)=\left[\begin{array}{c}
\beta_{i, 1}\left(y_{1}, \ldots, y_{i}, u\right) \\
\beta_{i, 2}\left(y_{1}, \ldots, y_{i}, u\right) \\
\vdots \\
\beta_{i, k_{i}}\left(y_{1}, \ldots, y_{i}, u\right)
\end{array}\right], C_{i}=\left[\begin{array}{lll}
0 & \cdots & 0
\end{array}\right]_{1 \times k_{i}}
$$

and

$$
A_{i}\left(y_{1}, \ldots, y_{i}, u\right)=\left[\begin{array}{cccc}
0 & \cdots & 0 & 0 \\
\alpha_{i, 1}\left(y_{1}, \ldots, y_{i}, u\right) & \cdots & 0 & 0 \\
\vdots & \ddots & \vdots & \vdots \\
0 & \cdots & \alpha_{i, k_{i}-1}\left(y_{1}, \ldots, y_{i}, u\right) & 0
\end{array}\right]
$$

with $\alpha_{i, j} \neq 0$ for all $y$ and $u$ in a certain studied neighborhood. For dynamical systems in the form 2, the high gain observer proposed in [4] can be applied directly (refer to [4] for more details).

In this paper, we will give the geometrical condition which is sufficient to guarantee the existence of a local diffeomorphism and an output injection to transform system (1) into the normal form (2). This kind of linearization will be named Multi-Output Dependent Observability normal form (MODO 
normal form) and generalizes the result obtained in $[5,19]$ for nonlinear systems with single output. In section 2 , we give some basic notations and a preliminary result is presented in Section 3 in order to introduce our method. In section 4, we propose our main result as a generalization of the result given in section 3 .

\section{Notations}

Consider system (1), with a possible reordering of $h_{i}$, we assume that there exist $k_{1} \geq k_{2} \geq \ldots \geq k_{m} \geq 1$ and $\sum_{i=1}^{m} k_{i}=n$ such that

$$
\theta=\left(\theta_{1}^{1}, \ldots, \theta_{1}^{k_{1}}, . ., \theta_{m}^{1}, . ., \theta_{m}^{k_{m}}\right)^{T}
$$

where

$$
\theta_{i}^{j}=d L_{f}^{j-1} h_{i}
$$

is a frame of the cotangent bundle $T^{*} U$. Thus, system (1) is observable. Integers $\left(k_{i}\right)_{1 \leq i \leq m}$ are called observability indices of system (1). For a nice description and more details about this assumption, see [11]. Obviously the list of these integers is generally not unique. For $1 \leq l \leq m$ and $1 \leq j \leq k_{l}$, let $\left(\tau_{i, 1}\right)_{1 \leq i \leq m}$ be the family of vector fields defined by:

$$
\theta_{l}^{j}\left(\tau_{i, 1}\right)=\left\{\begin{array}{l}
1, l=i, j=k_{i} \\
0, \text { otherwise }
\end{array}\right.
$$

and construct by induction the following family of vector fields:

$$
\tau_{i, r}=\left[\tau_{i, r-1}, f\right]=(-1)^{r-1} a d_{f}^{r-1} \tau_{i, r-1}, \text { for } 2 \leq r \leq k_{i} .
$$

The family $\tau=\left(\tau_{i, j}\right)_{1 \leq i \leq m}$ and $1 \leq j \leq k_{i}$ is a basis of the tangent bundle $T U$. The frame $T$ was addressed firstly in [10] for $p=0$ and $m=1$ and it is well-known in [10] that system (1) can be transformed into the normal form (2) if and only if we have

$$
\left[\tau_{1, i}, \tau_{1, j}\right]=0, \text { for } 1 \leq i, j \leq n .
$$

In this case, $\tau$ is a frame with which system (1) is in the form (2). 


\section{Preliminary results}

In this section, we will generalize the result stated in [5] and [19] to systems with multi-output.

Lemma 1 For a system in the form (2) we have for $1 \leq l \leq m, 1 \leq t \leq k_{l}$,

$$
\begin{aligned}
\tau_{l, t}= & \frac{1}{\pi_{l, t}} \frac{\partial}{\partial z_{l, t}}+\left(\begin{array}{c}
\sum_{r=1}^{l}\left(A_{r, t-1}^{l, t}\left(y_{1}, \ldots, y_{l}, u\right) z_{r, k_{r}-1}\right) \\
+\eta_{t-1}^{l, t}\left(y_{1}, \ldots, y_{l}, u\right)
\end{array}\right) \frac{\partial}{\partial z_{l, t-1}} \\
& +\sum_{i=1}^{t-2}\left(\sum_{r=1}^{l}\left(\begin{array}{c}
A_{r, i}^{l, t}\left(y_{1}, \ldots, y_{l}, u\right) z_{r, k_{r}-t+i} \\
+\sum_{j=k_{r}-t+i+1}^{k_{r}-1} \sum_{s=j}^{k_{r}-1} T_{r, j, s}^{l, t}\left(y_{1}, \ldots, y_{l}, u\right) z_{r, j} z_{r, s}
\end{array}\right)\right) \frac{\partial}{\partial z_{l, i}} \\
& +\sum_{i=1}^{t-2}\left(\begin{array}{c}
\sum_{r=1}^{l}\left(\begin{array}{c}
k_{r} \\
j=k_{r}-t+i+1
\end{array} \eta_{r, i}^{l, t}\left(y_{1}, \ldots, y_{l}, u\right) z_{r, j}\right) \\
+O_{\left(y_{1}, \ldots, y_{l}, u\right)}^{[3]}\left(\begin{array}{c}
z_{1, k_{1}-t+i+1}, . ., z_{1, k_{1}-1}, \\
\ldots, z_{l, k_{l}-t+i+1}, . ., z_{l, k_{l}-1}
\end{array}\right)
\end{array}\right) \frac{\partial}{\partial z_{l, i}},
\end{aligned}
$$

where $\pi_{l, k_{l}}=1$ and $\pi_{l, t-1}=\pi_{l, t} \alpha_{l, t-1}$ for $2 \leq t \leq k_{l}, \eta_{i}^{l, t}$ and $T_{r, j, q}^{l, t}$ are some smooth functions of $\left(y_{1}, \ldots, y_{l}, u\right), O_{\left(y_{1}, \ldots, y_{l}, u\right)}^{[3]}\left(\begin{array}{c}z_{1, k_{1}-t+i+1}, . ., z_{1, k_{1}-1}, \\ \ldots, z_{l, k_{l}-t+i+1}, . ., z_{l, k_{l}-1}\end{array}\right)$ represents the residue higher than order 2 with coefficient which is function of $\left(y_{1}, \ldots, y_{l}, u\right)$ and

$$
\begin{aligned}
& A_{r, i}^{l, t}\left(y_{1}, \ldots, y_{l}, u\right)=(-1)^{t-i+1} S_{r, t-i, 1}^{l, t} \frac{\partial_{y_{r}} \pi_{l, i}}{\pi_{l, i}^{2}} \pi_{r, k_{r}-t+i}+ \\
& \quad(-1)^{t-i+1} \sum_{m=t-i+1}^{t-1} S_{r, t-i, m-t+i+1}^{l, t} \frac{\partial_{y_{r}} \pi_{l, t-m}}{\pi_{l, t-m}^{2}}\left(\prod_{j=t-i+1}^{m} \alpha_{l, k-j}\right) \pi_{r, k_{r}-t+i},
\end{aligned}
$$

where $\partial_{y_{r}} \pi_{l, i}$ represents $\frac{\partial \pi_{l, i}\left(y_{1}, \ldots, y_{l}, u\right)}{\partial y_{r}}, S_{r, t-i, 1}^{l, t}$ and $S_{r, t-i, m-t+i+1}^{l, t}$ are defined as follows

$$
S_{r, j, 1}^{l, t}=1, S_{r, j, s}^{l, t}=S_{r, j-1, s}^{l, t-1}+S_{r, j, s-1}^{l, t-1}
$$

for $1 \leq l \leq m, 1 \leq r \leq l, 2 \leq t \leq k_{l}, 1 \leq j \leq t-1$ and $1 \leq s \leq t-j$.

Proof 1 For a system in the (2) form, for $1 \leq l \leq m, \tau_{l, 1}=\frac{1}{\pi_{l, 1}} \frac{\partial}{\partial z_{l, 1}}$, then we 
use equation (5) to obtain

$$
\begin{aligned}
\tau_{1, t}= & \frac{1}{\pi_{1, t}} \frac{\partial}{\partial z_{1, t}}+\left(A_{1, t-1}^{1, t}\left(y_{1}, u\right) z_{1, k_{1}-1}+\eta_{t-1}^{1, t}\left(y_{1}, u\right)\right) \frac{\partial}{\partial z_{1, t-1}} \\
& +\sum_{i=1}^{t-2}\left(A_{1, i}^{1, t}\left(y_{1}, u\right) z_{1, k_{1}-t+i}+\sum_{j=k_{1}-t+i+1}^{k_{1}-1} \sum_{l=j}^{k_{1}-1} T_{j, l}^{1, t}\left(y_{1}, u\right) z_{1, j} z_{1, l}\right) \frac{\partial}{\partial z_{1, i}} \\
& +\sum_{i=1}^{t-2}\left(\sum_{j=k_{1}-t+i+1}^{k_{1}} \eta_{i}^{1, t}\left(y_{1}, u\right) z_{1, j}+O_{\left(y_{1}, u\right)}^{[3]}\left(z_{1, k_{1}-t+i+1}, . ., z_{1, k_{1}-1}\right)\right) \frac{\partial}{\partial z_{1, i}},
\end{aligned}
$$

for $1 \leq t \leq k_{1}$ and

$$
\begin{aligned}
& \tau_{2, t}=\frac{1}{\pi_{2, t}} \frac{\partial}{\partial z_{2, t}}+\left(\begin{array}{c}
A_{1, t-1}^{2, t}\left(y_{1}, y_{2}, u\right) z_{1, k_{1}-1}+A_{2, t-1}^{2, t}\left(y_{1}, y_{2}, u\right) z_{2, k_{2}-1} \\
+\eta_{t-1}^{2, t}\left(y_{1}, y_{2}, u\right)
\end{array}\right) \frac{\partial}{\partial z_{2, t-1}} \\
& +\sum_{i=1}^{t-2}\left(\begin{array}{c}
A_{1, i}^{2, t}\left(y_{1}, y_{2}, u\right) z_{1, k_{1}-t+i} \\
+\sum_{j=k_{2}-t+i+1}^{k_{2}-1} \sum_{l=j}^{k_{2}-1} T_{1, j, l}^{2, t}\left(y_{1}, y_{2}, u\right) z_{1, j} z_{1, l}+ \\
A_{2, i}^{2, t}\left(y_{1}, y_{2}, u\right) z_{2, k_{2}-t+i} \\
+\sum_{j=k_{2}-t+i+1}^{k_{2}-1} \sum_{l=j}^{k_{2}-1} T_{2, j, l}^{2, t}\left(y_{1}, y_{2}, u\right) z_{2, j} z_{2, l}
\end{array}\right) \frac{\partial}{\partial z_{2, i}} \\
& +\sum_{i=1}^{t-2}\left(\begin{array}{c}
\sum_{j=k_{1}-t+i+1}^{k_{1}} \eta_{1, i}^{2, t}\left(y_{1}, y_{2}, u\right) z_{1, j}+\sum_{j=k_{2}-t+i+1}^{k_{2}} \eta_{2, i}^{2, t}\left(y_{1}, y_{2}, u\right) z_{2, j} \\
+O_{\left(y_{1}, y_{2}, u\right)}^{[3]}\left(z_{1, k_{1}-t+i+1}, . ., z_{1, k_{1}-1}, z_{2, k_{2}-t+i+1}, . ., z_{2, k_{2}-1}\right)
\end{array}\right) \frac{\partial}{\partial z_{2, i}},
\end{aligned}
$$

for $1 \leq t \leq k_{2}$. Then by an induction, for $1 \leq t \leq k_{l}$, we get

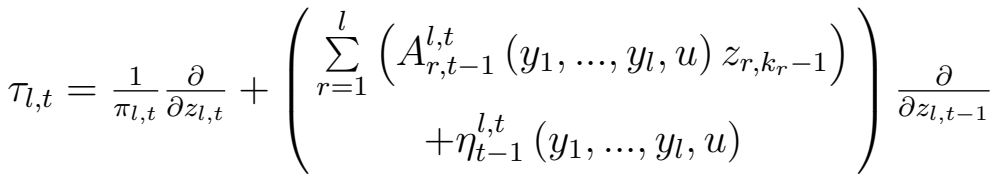

$$
\begin{aligned}
& +\sum_{i=1}^{t-2}\left(\sum_{r=1}^{l}\left(\begin{array}{c}
A_{r, i}^{l, t}\left(y_{1}, \ldots, y_{l}, u\right) z_{r, k_{r}-t+i} \\
+\sum_{j=k_{r}-t+i+1}^{k_{r}-1} \sum_{s=j}^{k_{r}-1} T_{r, j, s}^{l, t}\left(y_{1}, \ldots, y_{l}, u\right) z_{r, j} z_{r, s}
\end{array}\right)\right) \frac{\partial}{\partial z_{l, i}} \\
& +\sum_{i=1}^{t-2}\left(\begin{array}{c}
\sum_{r=1}^{l}\left(\sum_{j=k_{r}-t+i+1}^{k_{r}} \eta_{r, i}^{l, t}\left(y_{1}, \ldots, y_{l}, u\right) z_{r, j}\right) \\
+O_{\left(y_{1}, \ldots, y_{l}, u\right)}^{[3]}\left(\begin{array}{c}
z_{1, k_{1}-t+i+1}, . ., z_{1, k_{1}-1}, \\
\ldots, z_{l, k_{l}-t+i+1}, . ., z_{l, k_{l}-1}
\end{array}\right)
\end{array}\right) \frac{\partial}{\partial z_{l, i}},
\end{aligned}
$$

where

$$
A_{r, i}^{l, t}\left(y_{1}, \ldots, y_{l}, u\right)=(-1)^{t-i+1}\left(S_{r, t-i, 1}^{l, t} \frac{\partial_{y_{r}} \pi_{l, i}}{\pi_{l, i}^{2}}+\right.
$$




$$
\left.\sum_{m=t-i+1}^{t-1} S_{r, t-i, m-t+i+1}^{l, t} \frac{\partial_{y_{r}} \pi_{l, t-m}}{\pi_{l, t-m}^{2}}\left(\prod_{j=t-i+1}^{m} \alpha_{l, k-j}\right)\right) \pi_{r, k_{r}-t+i}
$$

with the coefficients $S_{r, j, s}^{l, t}$ defined by 9 .

In order to determine the $\alpha_{i, j}\left(y_{1}, \ldots, y_{i}, u\right)$ for $1 \leq i \leq m, 1 \leq j \leq k_{i}-1$, we also impose that for $1 \leq i, l \leq m$,

$$
\frac{\partial}{\partial z_{i, j}} h_{l} \circ \phi=\left\{\begin{array}{l}
1, \text { if } l=i \text { and } j=k_{i}, \\
0, \text { otherwise. }
\end{array}\right.
$$

Now, we are ready to state a set of partial differential equations which enables us to compute functions $\alpha_{i, j}\left(y_{1}, \ldots, y_{i}, u\right)$ for $1 \leq i \leq m, 1 \leq j \leq k_{i}-1$.

Proposition 1 If there exists a diffeomorphism which transforms system (1) into form (2) then for $1 \leq l \leq m, 1 \leq t \leq k_{l}-1,1 \leq s \leq l-1$,

$$
\left\{\begin{array}{l}
{\left[\tau_{l, t}, \tau_{s, k_{s}}\right]=\lambda_{l, t}^{s} \tau_{l, t} \bmod \operatorname{span}\left\{\tau_{l, 1}, \ldots, \tau_{l, t-1}\right\}} \\
{\left[\tau_{l, t}, \tau_{l, k_{l}}\right]=\lambda_{l, t}^{l} \tau_{l, t}+G_{l, k_{l}, t}^{[1]}+R_{l, t}}
\end{array}\right.
$$

where

$$
G_{l, k_{l}, t}^{[1]}=\left[\sum_{i=1}^{t-1}\left(\frac{1}{\pi_{l, t}} T_{l, t, k_{l}-t+i}^{l, t} z_{l, k_{l}-t+i}\right) \frac{\partial}{\partial z_{l, i}}\right]+\frac{1}{\pi_{l, t}} T_{l, t, t}^{l, t} z_{l, t} \frac{\partial}{\partial z_{l, 2 t-k_{l}}}
$$

and

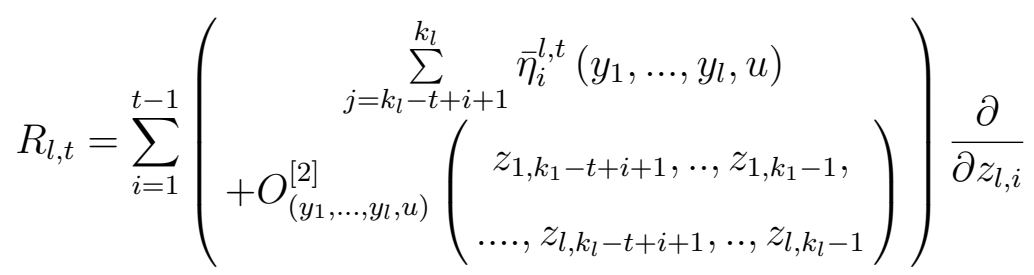

and

$$
\left\{\begin{array}{l}
\lambda_{l, t}^{s}=-\frac{\partial_{y_{s}} \pi_{l, t}}{\pi_{l, t}} \\
\lambda_{l, t}^{l}=\operatorname{diag}\left\{\delta_{1}^{l, t}\left(y_{1}, \ldots y_{l}, u\right), \ldots, \delta_{i}^{l, t}\left(y_{1}, \ldots y_{l}, u\right), \ldots, \delta_{t}^{l, t}\left(y_{1}, \ldots y_{l}, u\right), 0, \ldots, 0\right\}
\end{array}\right.
$$


defining $\partial_{y_{s}} \pi_{l, t}:=\frac{\partial\left[\pi_{l, t}\left(y_{1}, \ldots y_{l}, u\right)\right]}{\partial y_{s}}, \delta_{t}^{l, t}=A_{l, t}^{l, k_{l}}+\frac{\partial_{y_{l}} \pi_{l, t}}{\pi_{l, t}}$ and

$$
\delta_{i}^{l, t}=A_{l, i}^{l, k_{l}}-A_{l, k_{l}-t+i}^{l, k_{l}}-\frac{\partial_{y_{l}}\left(A_{l, i}^{l, t}\right)}{A_{l, i}^{l, t}}
$$

for $1 \leq i \leq t-1$, and $A_{l, i}^{l, t}$ is given in (8).

Proof 2 According to equation (7), for $1 \leq l \leq m, 1 \leq t \leq k_{l}-1$, we have

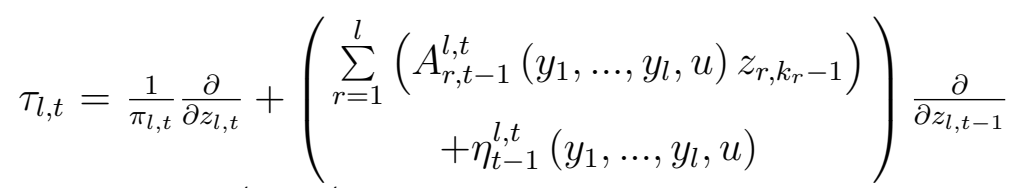

$$
\begin{aligned}
& +\sum_{i=1}^{t-2}\left(\sum_{r=1}^{l}\left(\begin{array}{c}
A_{r, i}^{l, t}\left(y_{1}, \ldots, y_{l}, u\right) z_{r, k_{r}-t+i} \\
+\sum_{j=k_{r}-t+i+1}^{k_{r}-1} \sum_{s=j}^{k_{r}-1} T_{r, j, s}^{l, t}\left(y_{1}, \ldots, y_{l}, u\right) z_{r, j} z_{r, s}
\end{array}\right)\right) \frac{\partial}{\partial z_{l, i}} \\
& +\sum_{i=1}^{t-2}\left(\begin{array}{c}
\sum_{r=1}^{l}\left(\sum_{j=k_{r}-t+i+1}^{k_{r}} \eta_{r, i}^{l, t}\left(y_{1}, \ldots, y_{l}, u\right) z_{r, j}\right) \\
+O_{\left(y_{1}, \ldots, y_{l}, u\right)}^{[3]}\left(\begin{array}{c}
z_{1, k_{1}-t+i+1}, . ., z_{1, k_{1}-1}, \\
\ldots, z_{l, k_{l}-t+i+1}, . ., z_{l, k_{l}-1}
\end{array}\right)
\end{array}\right) \frac{\partial}{\partial z_{l, i}},
\end{aligned}
$$

then we can calculate

$$
\left[\tau_{l, t}, \tau_{s, k_{s}}\right]=-\frac{\partial_{y_{s}} \pi_{l, t}}{\pi_{l, t}} \frac{\partial}{\partial z_{l, t}} \bmod \operatorname{span}\left\{\tau_{l, 1}, \ldots, \tau_{l, t-1}\right\}
$$

Defining $\lambda_{l, t}^{s}=-\frac{\partial_{y_{s}} \pi_{l, t}}{\pi_{l, t}}$, we obtain

$$
\left[\tau_{l, t}, \tau_{s, k_{s}}\right]=\lambda_{l, t}^{s} \tau_{l, t} \bmod \operatorname{span}\left\{\tau_{l, 1}, \ldots, \tau_{l, t-1}\right\}
$$

Moreover, as

$$
\begin{aligned}
{\left[\tau_{l, t}, \tau_{l, k_{l}}\right]=} & \left(A_{l, t}^{l, k_{l}}+\frac{\partial_{y_{l}} \pi_{l, t}}{\pi_{l, t}}\right) \frac{1}{\pi_{l, t}} \frac{\partial}{\partial z_{l, t}} \\
& +\sum_{i=1}^{t-1}\left(\begin{array}{c}
\left(A_{l, i}^{l, k_{l}}-A_{l, k_{l}-t+i}^{l, k_{l}}-\frac{\partial_{y_{l}}\left(A_{l, t}^{l, t}\right)}{A_{l, i}^{l, t}}\right) A_{l, i}^{l, t} z_{l, k_{l}-t+i} \\
+\frac{1}{\pi_{l, t}} T_{l, t, k_{l}-t+i}^{l, t} z_{l, k_{l}-t+i}
\end{array}\right) \frac{\partial}{\partial z_{l, i}} \\
& +\frac{1}{\pi_{l, t}} T_{l, t, t}^{l, t} z_{l, t} \frac{\partial}{\partial z_{l, 2 t-k_{l}}}
\end{aligned}
$$




$$
+\sum_{i=1}^{t-1}\left(\begin{array}{c}
\sum_{j=k_{l}-t+i+1}^{k_{l}} \bar{\eta}_{i}^{l, t}\left(y_{1}, \ldots, y_{l}, u\right) \\
+O_{\left(y_{1}, \ldots, y_{l}, u\right)}^{[2]}\left(\begin{array}{c}
z_{1, k_{1}-t+i+1}, . ., z_{1, k_{1}-1}, \\
\ldots, z_{l, k_{l}-t+i+1}, . ., z_{l, k_{l}-1}
\end{array}\right)
\end{array}\right) \frac{\partial}{\partial z_{l, i}}
$$

Set

$$
\lambda_{l, t}^{l}\left(y_{1}, \ldots, y_{l}, u\right)=\operatorname{diag}\left\{\delta_{1}^{l, t}\left(y_{1}, \ldots, y_{l}, u\right), \ldots, \delta_{i}^{l, t}\left(y_{1}, \ldots, y_{l}, u\right), \ldots, \delta_{t}^{l, t}\left(y_{1}, \ldots, y_{l}, u\right), \ldots, 0, \ldots, 0\right\},
$$

where $\delta_{t}^{l, t}=A_{l, t}^{l, k_{l}}+\frac{\partial_{y_{l}} \pi_{l, t}}{\pi_{l, t}}$ and

$$
\delta_{i}^{l, t}=A_{l, i}^{l, k_{l}}-A_{l, k_{l}-t+i}^{l, k_{l}}-\frac{\partial_{y_{l}}\left(A_{l, i}^{l, t}\right)}{A_{l, i}^{l, t}}
$$

for $1 \leq i \leq t-1$, then

$$
\left[\tau_{l, t}, \tau_{l, k_{l}}\right]=\lambda_{l, t}^{l} \tau_{l, t}+G_{l, k_{l}, t}^{[1]}+R_{l, t}
$$

Remark 1 In equation (11), $\lambda_{l, t}^{l}$ could be uniquely determined since $G_{l, k_{l}, t}^{[1]}$ might be separated according to the coefficients of second-order terms in $\tau_{l, k_{l}}$.

Finally, the following result enables us to determine all the functions $\alpha_{l, i}\left(y_{1}, \ldots, y_{l}, u\right)$ for $1 \leq l \leq m$ and $1 \leq i \leq k_{l}-1$.

Proposition 2 If there exists a diffeomorphism which transforms system (1) into form (2), then $\alpha_{l, i}=\frac{\pi_{l, i}}{\pi_{l, i+1}}$ for $1 \leq l \leq m$ and $1 \leq i \leq k_{l}-2$, and $\alpha_{l, k_{l}-1}=\pi_{l, k_{l}-1}$, where the $\pi_{l, i}$ for $1 \leq i \leq k_{l}-1$ is the solution of the following partial differential equations:

$$
\left\{\begin{array}{l}
\frac{\partial_{y_{s}} \pi_{l, i}}{\pi_{l, i}}=-\lambda_{l, i}^{s}\left(y_{1}, \ldots, y_{l}\right), \text { for } 1 \leq s \leq l-1 \text { and } 1 \leq i \leq k_{l}-1 \\
\frac{\partial_{y_{l}} \pi_{l, i}}{\pi_{l, i}}=\exp \int\left(\delta_{i}^{l, i}-\delta_{i}^{l, k_{l}-1}-\delta_{i+1}^{l, i+1}\right) d y_{l}-\bar{B}_{l, i}^{l, k_{l}-1}, \text { for } 1 \leq i \leq k_{l}-2 \\
\frac{\partial_{y_{l}} \pi_{l, k_{l}-1}}{\pi_{l, k_{l}-1}}=\frac{\delta_{k_{l}-1}^{l, k_{l}-1}-\bar{A}_{l, k_{l}-1}^{l, k_{l}}}{2}
\end{array}\right.
$$

with $\bar{B}_{l, 1}^{l, 0}=0$ and for $1 \leq i, t \leq k_{l}-1$

$$
\bar{B}_{l, i}^{l, t}=\sum_{m=t-i+1}^{t-1} S_{t-i, m-t+i+1}^{l, t} \frac{\partial_{y_{l}} \pi_{l, t-m}}{\pi_{l, t-m}} .
$$

Proof 3 Obviously, according to (10), we have

$$
\frac{\partial_{y_{s}} \pi_{l, i}}{\pi_{l, i}}=-\lambda_{l, i}^{s}\left(y_{1}, \ldots, y_{l}, u\right), \text { for } 1 \leq s \leq l-1 \text { and } 1 \leq i \leq k_{l}-1
$$


Define

$$
B_{l, i}^{l, t}=\frac{\partial_{y_{l}} \pi_{l, i}}{\pi_{l, i}}+\bar{B}_{l, i}^{l, t}
$$

where $1 \leq l \leq m$ and $1 \leq i, t \leq k_{l}-1$

According to (8), for $1 \leq i, k \leq k_{l}-1$,

$$
\frac{\partial_{y_{l}}\left(A_{l, i}^{l, t}\right)}{A_{l, i}^{l, t}}=\frac{\partial_{y_{l}}\left(B_{l, i}^{l, t}\right)}{B_{l, i}^{l, t}}-\frac{\partial_{y_{l}} \pi_{l, i}}{\pi_{l, i}}+\frac{\partial_{y_{l}} \pi_{l, k_{l}-t+i}}{\pi_{l, k_{l}-t+i}} .
$$

As $\delta_{t}^{l, t}=A_{l, t}^{l, k_{l}}+\frac{\partial_{y_{l}} \pi_{l, t}}{\pi_{l, t}}$, hence

$$
\begin{aligned}
\delta_{i}^{l, k_{l}-1} & =A_{l, i}^{l, k_{l}}-A_{l, 1+i}^{l, k_{l}}-\frac{\partial_{y_{l}}\left(B_{l, i}^{l, k_{l}-1}\right)}{B_{l, i}^{l, k_{l}-1}}+\frac{\partial_{y_{l}} \pi_{l, i}}{\pi_{l, i}}-\frac{\partial_{y_{l}} \pi_{l, 1+i}}{\pi_{l, 1+i}} \\
& =\delta_{i}^{l, i}-\delta_{1+i}^{l, 1+i}-\partial_{y_{l}}\left(\frac{\partial_{y_{l}} \pi_{l, i}}{\pi_{l, i}}+\bar{B}_{l, i}^{l, k_{l}-1}\right) /\left(\frac{\partial_{y_{l}} \pi_{l, i}}{\pi_{l, i}}+\bar{B}_{l, i}^{l, k_{l}-1}\right) .
\end{aligned}
$$

which yields

$$
\frac{\partial_{y_{l}} \pi_{l, i}}{\pi_{l, i}}=\exp \int\left(\delta_{i}^{l, i}-\delta_{i}^{l, k_{l}-1}-\delta_{i+1}^{l, i+1}\right) d y_{l}-\bar{B}_{l, i}^{l, k_{l}-1}, \text { for } 1 \leq i \leq k_{l}-2
$$

where $\bar{B}_{l, i}^{l, k_{l}-1}$ is defined in (13) and $c_{l, i} \neq 0$. As $\delta_{k_{l}-1}^{l, k_{l}-1}=2 \frac{\partial_{y_{l}} \pi_{l, k_{l}-1}}{\pi_{l, k_{l}-1}}+\bar{A}_{l, k_{l}-1}^{l, k_{l}}$, where $\bar{A}_{l, k_{l}-1}^{l, k_{l}}=\sum_{m=2}^{k_{l}-1} S_{1, m}^{l, k_{l}} \frac{\partial_{y_{l}} \pi_{l, k_{l}-m}}{\pi_{l, k_{l}-m}}$, then we obtain

$$
\frac{\partial_{y_{l}} \pi_{l, k_{l}-1}}{\pi_{l, k_{l}-1}}=\frac{\delta_{k_{l}-1}^{l, k_{l}-1}-\bar{A}_{l, k_{l}-1}^{l, k_{l}}}{2}
$$

\section{Main result}

If there exists a diffeomorphism which transforms system (1) into form (2), then equation (12) of Proposition 2 gives all $\alpha_{l, i}\left(y_{1}, \ldots, y_{l}, u\right)$ for $1 \leq l \leq m$ and $1 \leq i \leq k_{l}-1$. Therefore, let us consider a new family of vector fields defined as follows:

$$
\widetilde{\tau}_{l, 1}=\pi_{l, 1}\left(y_{1}, \ldots, y_{l}, u\right) \tau_{l, 1}
$$




$$
\widetilde{\tau}_{l, i}=\frac{1}{\alpha_{l, i-1}}\left[\tau_{l, i-1}, f\right] \text { for } i=2: k_{l}
$$

where $\pi_{l, 1}\left(y_{1}, \ldots, y_{l}, u\right)=\prod_{i=1}^{k_{l}-1} \alpha_{l, i}\left(y_{1}, \ldots, y_{l}, u\right)$. Set $\widetilde{\tau}=\left(\widetilde{\tau}_{i, j}\right)_{1 \leq i \leq m}$ and $1 \leq j \leq k_{i}$ and $\widetilde{\Lambda}=\theta \widetilde{\tau}$, we can define the following multi 1 -form:

$$
\omega=\widetilde{\Lambda}^{-1} \theta
$$

It is clear that

$$
\omega \widetilde{\tau}=I_{n \times n}
$$

Then we are ready to state our main result.

Theorem 1 There exists a diffeomorphism which transforms system (1) into a MODO normal form (2) if

i) there exists a family of functions $\alpha_{l, i}\left(y_{1}, \ldots, y_{l}, u\right)$ for $1 \leq l \leq m$ and $1 \leq i \leq$ $k_{l}-1$ such that the family of vector fields $\widetilde{\tau}_{l, i}$ for $1 \leq l \leq m$ and $1 \leq i \leq k_{l}-1$ defined in (15) satisfies the following commutativity conditions

$$
\left[\widetilde{\tau}_{i, j}, \widetilde{\tau}_{s, l}\right]=0, \text { for } 1 \leq i, s \leq m, 1 \leq j \leq k_{i} \text { and } 1 \leq l \leq k_{s}
$$

or

ii) there exists a family of functions $\alpha_{l, i}\left(y_{1}, \ldots, y_{l}, u\right)$ for $1 \leq l \leq m$ and $1 \leq i \leq k_{l}-1$ such that the $R^{n}$-valued form $\omega$ defined in (16) satisfies the following condition

$$
d \omega=0
$$

Proof 4 Assume that there exist $\alpha_{l, i}\left(y_{1}, \ldots, y_{l}, u\right)>0$ for $1 \leq l \leq m$ and $1 \leq$ $i \leq k_{l}-1$ such that $\left[\widetilde{\tau}_{i, j}, \widetilde{\tau}_{s, l}\right]=0$ for $1 \leq i, s \leq m, 1 \leq j \leq k_{i}$ and $1 \leq l \leq k_{s}$, then it is well-known ([12], [9]) that we can find a local diffeomorphism $\phi=z$ such that

$$
\phi_{*}\left(\widetilde{\tau}_{l, i}\right)=\frac{\partial}{\partial z_{l, i}}
$$

As $\phi_{*}\left(\widetilde{\tau}_{l, i}\right)=\frac{\partial}{\partial z_{l, i}}$ is constant, hence

$$
\frac{\partial}{\partial z_{l, i}} \phi_{*}(f)=\phi_{*}\left(\left[\widetilde{\tau}_{l, i}, f\right]\right)=\alpha_{l, i} \phi_{*}\left(\widetilde{\tau}_{l, i+1}\right)=\alpha_{l, i} \frac{\partial}{\partial z_{l, i+1}},
$$


thus $\frac{\partial}{\partial z_{l, i}} \phi_{*}(f)=\alpha_{l, i} \frac{\partial}{\partial z_{l, i+1}}$ for $1 \leq l \leq m$ and $1 \leq i \leq k_{l}-1$. Consequently, by integration we obtain: $\phi_{*}(f)=A(y, u) z+\beta(y, u)$. Moreover, as dh० $\widetilde{\tau}_{l, i}=0$ for $1 \leq l \leq m$ and $1 \leq i \leq k_{l}-1$ and $d h \circ \widetilde{\tau}_{l, k_{l}-1}=1$, we obtain $h \circ \phi=C z$ where $C$ is defined in (1).

Finally, in order to prove that in Theorem 1 Condition i) is equivalent to Condition ii), it is sufficient to prove that equation (17) is equivalent to equation (18). Recall that for any two vector fields $X, Y$, we have

$$
d \omega(X, Y)=L_{X}(\omega(Y))-L_{Y}(\omega(X))-\omega([X, Y]) .
$$

Setting $X=\widetilde{\tau}_{i . j}$ and $Y=\widetilde{\tau}_{s . l}$, we obtain

$$
d \omega\left(\widetilde{\tau}_{i, j}, \widetilde{\tau}_{s, l}\right)=L_{\widetilde{\tau}_{i, j}} \omega\left(\widetilde{\tau}_{s, l}\right)-L_{\widetilde{\tau}_{s, l}} \omega\left(\widetilde{\tau}_{i, j}\right)-\omega\left(\left[\widetilde{\tau}_{i, j}, \widetilde{\tau}_{s, l}\right]\right) .
$$

As $\omega\left(\widetilde{\tau}_{s, l}\right)$ and $\omega\left(\widetilde{\tau}_{i, j}\right)$ are constant, then we have

$$
d \omega\left(\widetilde{\tau}_{i, j}, \widetilde{\tau}_{s, l}\right)=-\omega\left(\left[\widetilde{\tau}_{i, j}, \widetilde{\tau}_{s, l}\right]\right)
$$

Because $\omega$ is an isomorphism and $\left(\widetilde{\tau}_{i, j}\right)_{1 \leq i \leq m}$ and $1 \leq j \leq k_{l}-1$ is a basis of $T U$, then equation (17) is equivalent to equation (18).

Example 1 Let us consider the following system:

$$
\left\{\begin{array}{l}
\dot{x}_{1,1}=\frac{\gamma\left(y_{1}, u\right)}{1+x_{3}} x_{1,1} x_{1,2}, \\
\dot{x}_{1,2}=\frac{\mu\left(y_{1}, u\right)}{1+x_{1,3}} x_{1,1}, \\
\dot{x}_{1,3}=\gamma\left(y_{1}, u\right) x_{1,2}, \\
\dot{x}_{2,1}=\beta\left(y_{1}, y_{2}, u\right) \frac{x_{2,1}^{2}}{\left(1+x_{2,2}\right)^{2}} \\
\dot{x}_{2,2}=\beta\left(y_{1}, y_{2}, u\right) \frac{x_{2,1}}{\left(1+x_{2,2}\right)} \\
y_{1}=x_{1,3}, \\
y_{2}=x_{2,2},
\end{array}\right.
$$

with $\left.y_{1} \in\right]-1,1\left[, y_{2} \in\right]-1,1\left[\right.$ and $\gamma \mu \beta \neq 0$ for any $u, y_{1}$ and $y_{2}$, then we can 


\section{obtain}

$$
\left\{\begin{aligned}
\tau_{1,1} & =\frac{1+x_{1,3}}{\gamma \mu} \frac{\partial}{\partial x_{1,1}}, \\
\tau_{1,2}= & \frac{1}{\gamma\left(y_{1}, u\right)} \frac{\partial}{\partial x_{1,2}}+\left(\left(1+x_{1,3}\right) \frac{\partial_{y_{1}}\left(\gamma\left(y_{1}, u\right) \mu\left(y_{1}, u\right)\right)}{\gamma\left(y_{1}, u\right) \mu^{2}\left(y_{1}, u\right)}\right) x_{1,2} \frac{\partial}{\partial x_{1,1}}, \\
\tau_{1,3}= & \frac{\partial}{\partial x_{1,3}}+\left(\frac{\partial y_{1}\left(\mu\left(y_{1}, u\right) \gamma\left(y_{1}, u\right)\right)}{\left(\mu\left(y_{1}, u\right) \gamma\left(y_{1}, u\right)\right)}+\frac{\partial_{y_{1}} \gamma\left(y_{1}, u\right)}{\gamma\left(y_{1}, u\right)}\right) x_{1,2} \frac{\partial}{\partial x_{1,2}} \\
& +\left(\frac{1}{1+x_{1,3}}-\frac{\partial_{y_{1}}\left(\gamma\left(y_{1}, u\right) \mu\left(y_{1}, u\right)\right)}{\gamma\left(y_{1}, u\right) \mu\left(y_{1}, u\right)}\right) x_{1,1} \frac{\partial}{\partial x_{1,1}} \\
\tau_{2,1}= & \frac{\left(1+x_{2,2}\right)}{\beta\left(y_{1}, y_{2}, u\right)} \frac{\partial}{\partial x_{2,1}} \\
\tau_{2,2}= & \frac{\partial}{\partial x_{2,2}}+\left(\begin{array}{c}
2 \frac{x_{2,1}}{\left(1+x_{2,2}\right)}-\partial_{y_{1}}\left(\frac{\left(1+x_{2,2}\right)}{\beta\left(y_{1}, y_{2}, u\right)}\right) \gamma\left(y_{1}, u\right) x_{2,2} \\
-\partial_{y_{2}}\left(\frac{\left(1+x_{2,2}\right)}{\beta\left(y_{1}, y_{2}, u\right)}\right) \beta\left(y_{1}, y_{2}, u\right) \frac{x_{2,1}}{\left(1+x_{2,2}\right)}
\end{array}\right) \frac{\partial}{\partial x_{2,1}}
\end{aligned}\right.
$$

which yields

$$
\left\{\begin{aligned}
\delta_{1,1}^{1} & =0 \\
\delta_{2,2}^{1} & =2 \frac{\partial_{y_{1}} \gamma\left(y_{1}, u\right)}{\gamma\left(y_{1}, u\right)}+\frac{\partial_{y_{1}}\left(\mu\left(y_{1}, u\right) \gamma\left(y_{1}, u\right)\right)}{\left(\mu\left(y_{1}, u\right) \gamma\left(y_{1}, u\right)\right)} \\
\delta_{2,1}^{1} & =-\frac{\partial_{y_{1}}\left(\mu\left(y_{1}, u\right) \gamma\left(y_{1}, u\right)\right)}{\left(\mu\left(y_{1}, u\right) \gamma\left(y_{1}, u\right)\right)}-2 \frac{\partial_{y_{1}} \gamma\left(y_{1}, u\right)}{\gamma\left(y_{1}, u\right)} \\
& -\partial_{y_{1}}\left(\frac{\partial_{y_{1}}\left(\mu\left(y_{1}, u\right) \gamma\left(y_{1}, u\right)\right)}{\left(\mu\left(y_{1}, u\right) \gamma\left(y_{1}, u\right)\right)}\right) /\left(\frac{\partial_{y_{1}}\left(\mu\left(y_{1}, u\right) \gamma\left(y_{1}, u\right)\right)}{\left(\mu\left(y_{1}, u\right) \gamma\left(y_{1}, u\right)\right)}\right) \\
\lambda_{1,1}^{1} & =-\frac{\partial_{y_{1}} \beta\left(y_{1}, y_{2}, u\right)}{\beta\left(y_{1}, y_{2}, u\right)} \\
\delta_{1,1}^{2} & =2 \frac{\partial_{y_{2}} \beta\left(y_{1}, y_{2}, u\right)}{\beta\left(y_{1}, y_{2}, u\right)}
\end{aligned}\right.
$$

Then, according to (12), we have

$$
\left\{\begin{array}{l}
\frac{\partial y_{1} \pi_{11}}{\pi_{11}}=\exp \int\left(\delta_{1,1}^{1}-\delta_{1,2}^{1}-\delta_{2,2}^{1}\right) d y_{1} \\
\frac{\partial y_{2} \pi_{12}}{\pi_{12}}=\frac{1}{2}\left(\delta_{2,2}^{1}-\frac{\partial_{y_{1}} \pi_{11}}{\pi_{11}}\right) \\
\frac{\partial_{y_{1}} \pi_{21}}{\pi_{21}}=-\lambda_{1,1}^{1}=\frac{\partial_{y_{1}} \beta\left(y_{1}, y_{2}, u\right)}{\beta\left(y_{1}, y_{2}, u\right)} \\
\frac{\partial_{y_{2}} \pi_{21}}{\pi_{21}}=\frac{1}{2} \delta_{2,1}^{2}=\frac{\partial_{y_{2}} \beta\left(y_{1}, y_{2}, u\right)}{\beta\left(y_{1}, y_{2}, u\right)}
\end{array}\right.
$$

which gives us $\pi_{11}=c_{1} \gamma\left(y_{1}, u\right) \mu\left(y_{1}, u\right), \pi_{12}=c_{2} \gamma\left(y_{1}, u\right)$ and $\pi_{2,1}=\alpha_{2,1}=$ $c_{3} \beta\left(y_{1}, y_{2}, u\right)$, then we can obtain $\alpha_{1,1}\left(y_{1}, u\right)=\frac{c_{1}}{c_{2}} \mu\left(y_{1}, u\right)$ and $\alpha_{1,2}=c_{2} \gamma\left(y_{1}, u\right)$. Then we have

$$
\left\{\begin{array}{l}
\widetilde{\tau}_{1,1}=c_{1}\left(1+x_{1,3}\right) \frac{\partial}{\partial x_{1,1}} \\
\widetilde{\tau}_{1,2}=c_{2} \frac{\partial}{\partial x_{1,2}}, \\
\widetilde{\tau}_{1,3}=\frac{\partial}{\partial x_{1,3}}+\frac{x_{1,1}}{1+x_{1,3}} \frac{\partial}{\partial x_{1,1}} .
\end{array}\right.
$$


and

$$
\left\{\begin{array}{l}
\tilde{\tau}_{2,1}=c_{3}\left(1+x_{2,2}\right) \frac{\partial}{\partial x_{2,1}} \\
\tilde{\tau}_{2,2}=\frac{\partial}{\partial x_{2,2}}+\frac{x_{2,1}}{1+x_{2,2}} \frac{\partial}{\partial x_{2,1}}
\end{array}\right.
$$

It is obvious $\left[\tilde{\tau}_{i j}, \tilde{\tau}_{s t}\right]=0$ for $1 \leq i, s \leq 2, j \in[1,3], t \in[1,2]$. So we have

$$
\omega=\Lambda^{-1} \tilde{\tau}=\left[\begin{array}{ccccc}
\frac{1}{c_{1}\left(1+x_{1,3}\right)} & 0 & -\frac{1}{c_{1}\left(1+x_{1,3}\right)^{2}} x_{1,1} & 0 & 0 \\
0 & \frac{1}{c_{2}} & 0 & 0 & 0 \\
0 & 0 & 1 & 0 & 0 \\
0 & 0 & 0 & \frac{1}{c_{3}\left(1+x_{2,2}\right)}-\frac{x_{2,1}}{c_{3}\left(1+x_{2,2}\right)^{2}} \\
0 & 0 & 0 & 0 & 1
\end{array}\right]
$$

which yields

$$
z=\phi(x)=\left(\frac{x_{1,1}}{c_{1}\left(1+x_{1,3}\right)}, \frac{1}{c_{2}} x_{1,2}, x_{1,3}, \frac{x_{2,1}}{c_{3}\left(1+x_{2,2}\right)}, x_{2,2}\right)^{T}
$$

with this diffeomorphism, system (19) is transformed into:

$$
\left\{\begin{array}{l}
\dot{z}_{1,1}=0, \\
\dot{z}_{1,2}=\frac{c_{1}}{c_{2}} \mu\left(y_{1}, u\right) z_{1,1}, \\
\dot{z}_{1,3}=c_{2} \gamma\left(y_{1}, u\right) z_{1,2}, \\
\dot{z}_{2,1}=0 \\
\dot{z}_{2,2}=c_{3} \beta\left(y_{1}, y_{2}, u\right) z_{2,1} \\
y_{1}=z_{1,3}, \\
y_{2}=z_{2,2} .
\end{array}\right.
$$

\section{Extension to system with unknown inputs}

In this section, we extend our results to a system with unknown inputs and which is in following form:

$$
\left\{\begin{array}{l}
\dot{x}=f(x, u)+g(x, u, w) \\
y=\left(h_{1}(x), \ldots, h_{m}(x)\right)^{T}
\end{array}\right.
$$


where $x \in R^{n}, u \in R^{p}, w \in R^{q}, f: R^{n} \times R^{p} \rightarrow R^{n}, g: R^{n} \times R^{p} \times R^{q} \rightarrow R^{n}$ and $h: R^{n} \rightarrow R^{m}$ are analytic functions. For system (20) we seek the MODO normal form along its output trajectory as follows:

$$
\left\{\begin{array}{l}
\dot{z}=A(y, u) z+\beta(y, u)+\eta(y, u, w) \\
y=C z
\end{array}\right.
$$

where $A(y, u), \beta(y, u)$ and $C$ are defined in (2), and the matrix $\eta(y, u, w)$ is defined as the matrix $\beta(y, u)$.

Theorem 2 System (20) can be transformed into MODO normal form (21) by a diffeomorphism and an output injection if and only if there is a family functions $\left(\alpha_{i, j}(y, u)\right)_{1 \leq i \leq m}$ and ${ }_{1 \leq j \leq k_{i}-1}$, satisfies both commutativity conditions:

i) For $1 \leq i, s \leq m, 1 \leq j \leq k_{i}$ and $1 \leq l \leq k_{s}$,

$$
\left[\widetilde{\tau}_{i, j}, \widetilde{\tau}_{s, l}\right]=0
$$

ii)

$$
\left[g, \widetilde{\tau}_{i, j}\right]=0 \text { for } 1 \leq i \leq m, 1 \leq j \leq k_{i}-1
$$

Proof 5 From Theorem 1, we can state that there exists a diffeomorphism $\phi$ such that

$$
\phi_{*}(f)=A(y, u) z+\beta(y, u) .
$$

For $1 \leq i \leq m$ and $1 \leq j \leq k_{l}-1$, because $\phi_{*}\left(\widetilde{\tau}_{i, j}\right)=\frac{\partial}{\partial z_{i, j}}$ is constant, hence we have

$$
\frac{\partial}{\partial z_{i, j}} \phi_{*}(g)=\phi_{*}\left(\left[g, \widetilde{\tau}_{i, j}\right]\right)=0
$$

Therefore $\phi_{*}(g)=\eta(y, u, w)$. Thus, we obtain the form (21).

Remark 2 If $g(x, u, w)=g_{1}(x, u) w_{1}+. .+g_{q}(x, u) w_{q}$, and also both conditions i) and $i$ ) of Theorem 2 are fulfilled, then:

$$
\eta(y, u, w)=B_{1}(y, u) w_{1}+. .+B_{q}(y, u) w_{q}
$$

Let us now study some special cases of the output injection.

Corollary 1 Let us assume that conditions i) and ii) of theorem2 are fulfilled: 
a) if $\left[g, \widetilde{\tau}_{i, k_{i}}\right]=0$, for $1 \leq i \leq m$, then:

$$
\eta(y, u, w)=\eta(u, w)
$$

b) if $g(x, u, w)=g_{1}(x, u) u_{1}+. .+g_{q}(x, u) u_{q}$ and

$$
\left[g_{k}, \widetilde{\tau}_{i, k_{i}}\right]=0 \text { for } 1 \leq i \leq m, 1 \leq k \leq p
$$

then

$$
\eta(y, u, w)=B_{1}(u) w_{1}+. .+B_{q}(u) w_{q} .
$$

where $B_{i}$ are functions of $u$. c) if $g(x, w)=g_{1}(x) w_{1}+. .+g_{q}(x) w_{q}$ and

$$
\left[g_{k}, \widetilde{\tau}_{i, k_{i}}\right]=0 \text { for } 1 \leq i \leq m, 1 \leq k \leq q
$$

then

$$
\eta(y, u, w)=B_{1} w_{1}+. .+B_{q} w_{p}
$$

where $B_{i}$ are constant vector fields.

Example 2 Let us consider the following system

$$
\left\{\begin{array}{l}
\dot{x}_{1,1}=\gamma\left(y_{1}, u\right) \frac{x_{1,1}^{2}}{\left(1+x_{1,2}\right)^{2}}+\frac{x_{1,1}}{\left(1+x_{1,2}\right)} w_{1} \\
\dot{x}_{1,2}=\gamma\left(y_{1}, u\right) \frac{x_{1,1}}{1+x_{1,2}}+w_{1}+x_{2,1} w_{2} \\
\dot{x}_{2,1}=\beta\left(y_{1}, y_{2}, u\right) x_{1,2} \\
y_{1}=x_{1,2} \\
y_{2}=x_{2,1}
\end{array}\right.
$$

with $\left.y_{1} \in\right]-1,1\left[, \gamma \beta \neq 0\right.$ for any $u, y_{1}$ and $y_{2}$, then we can obtain

$$
\left\{\begin{array}{l}
\tau_{1,1}=\frac{1+x_{1,2}}{\gamma\left(y_{1}, u\right)} \frac{\partial}{\partial x_{1,1}} \\
\tau_{1,2}=\frac{\partial}{\partial x_{1,2}}+\left(3 \gamma^{2}\left(y_{1}, u\right) \frac{x_{1,1}}{\left(1+x_{1,2}\right)^{3}}-\gamma\left(y_{1}, u\right) \partial_{y_{1}}\left(\gamma\left(y_{1}, u\right)\right) \frac{x_{1,1}}{\left(1+x_{1,2}\right)^{2}}\right) \frac{\partial}{\partial x_{1,1}} \\
\tau_{2,1}=\frac{\partial}{\partial x_{2,1}}
\end{array}\right.
$$

which yields

$$
\delta_{1}^{1,1}=2 \frac{\partial_{y_{1}} \gamma\left(y_{1}, u\right)}{\gamma\left(y_{1}, u\right)}
$$


so according to equation (12), we obtain

$$
\frac{\partial_{y_{1}} \pi_{1,1}}{\pi_{1,1}}=\frac{\delta_{1}^{1,1}}{2}=\frac{\partial_{y_{1}} \gamma\left(y_{1}, u\right)}{\gamma\left(y_{1}, u\right)}
$$

which gives $\pi_{1,1}=\alpha_{1,1}=c_{1} \gamma\left(y_{1}, u\right)$. Consequently, we have

$$
\left\{\begin{array}{l}
\widetilde{\tau}_{1,1}=c_{1}\left(1+x_{1,2}\right) \frac{\partial}{\partial x_{1,1}} \\
\widetilde{\tau}_{1,2}=\frac{\partial}{\partial x_{1,2}}+\frac{x_{1,1}}{1+x_{1,2}} \frac{\partial}{\partial x_{2,1}} \\
\widetilde{\tau}_{2,1}=\frac{\partial}{\partial x_{2,1}}
\end{array}\right.
$$

As $g_{1}=\frac{\partial}{\partial x_{1,2}}+\frac{x_{1,1}}{1+x_{1,2}} \frac{\partial}{\partial x_{2,1}}=\widetilde{\tau}_{1,2}$, so $\left[g_{1}, \widetilde{\tau}_{1,1}\right]=\left[g_{1}, \widetilde{\tau}_{1,2}\right]=\left[g_{1}, \widetilde{\tau}_{2,1}\right]=0$. Moreover, due to $g_{2}=x_{2,1} \frac{\partial}{\partial x_{1,2}}$, then we have $\left[g_{2}, \widetilde{\tau}_{1,1}\right]=0,\left[g_{2}, \widetilde{\tau}_{1,2}\right] \neq 0$ and $\left[g_{2}, \widetilde{\tau}_{2,1}\right] \neq 0$. Thus system (24) can be putted into the following form:

$$
\left\{\begin{array}{l}
\dot{z}_{1,1}=-\frac{x_{1,1}}{c_{1}\left(1+x_{1,2}\right)^{2}} x_{2,1} w_{2} \\
\dot{z}_{1,2}=c_{1} \gamma\left(y_{1}, u\right) z_{1,1}+w_{1}+z_{2,1} w_{2} \\
\dot{z}_{2,1}=\beta\left(y_{1}, y_{2}, u\right) z_{1,2} \\
y_{1}=z_{1,2} \\
y_{2}=z_{2,1}
\end{array}\right.
$$

by the following diffeomorphism

$$
\phi=\left(\begin{array}{c}
\frac{x_{1,1}}{c_{1}\left(1+x_{1,2}\right)} \\
x_{1,2} \\
x_{2,1}
\end{array}\right)
$$

Corollary 2 Assume conditions $i$ ) and $i$ ) of theorem 2 are fulfilled and $m \geq$ $q$, then the OMC (Observability Matching Condition) for system (21) is as follows:

$$
\begin{aligned}
& \operatorname{rank} \frac{\partial}{\partial w} \eta_{i, 1}(y, u, w)=q \\
& \text { and } \\
& \frac{\partial}{\partial w} \eta_{i, j}(y, u, w)=0, \text { for } 1 \leq i \leq m, 2 \leq j \leq k_{i}
\end{aligned}
$$


Here we give another example to highlight the above corollary.

Example 3 Consider the following system:

$$
\left\{\begin{array}{l}
\dot{x}_{1,1}=\gamma\left(y_{1}, u\right) \frac{x_{1,1}^{2}}{\left(1+x_{1,2}\right)^{2}}+\gamma\left(y_{1}, u\right) w_{1}+w_{2} \\
\dot{x}_{1,2}=\gamma\left(y_{1}, u\right) \frac{x_{1,1}}{1+x_{1,2}} \\
\dot{x}_{2,1}=\beta\left(y_{1}, y_{2}, u\right) x_{1,2}+w_{1}+\beta\left(y_{1}, y_{2}, u\right) w_{2} \\
y_{1}=x_{1,2} \\
y_{2}=x_{2,1}
\end{array}\right.
$$

with $\left.y_{1} \in\right]-1,1\left[, \beta \gamma \neq 0\right.$ for any $u, y_{1}$ and $y_{2}$. According to example (24) we can get $\pi_{1,1}=\alpha_{1,1}=c_{1} \gamma\left(y_{1}, u\right)$ which yields the following new vector fields:

$$
\left\{\begin{array}{l}
\widetilde{\tau}_{1,1}=c_{1}\left(1+x_{1,2}\right) \frac{\partial}{\partial x_{1,1}} \\
\widetilde{\tau}_{1,2}=\frac{\partial}{\partial x_{1,2}}+\frac{x_{1,1}}{1+x_{1,2}} \frac{\partial}{\partial x_{2,1}} \\
\widetilde{\tau}_{2,1}=\frac{\partial}{\partial x_{2,1}}
\end{array}\right.
$$

which means the diffeomorphism is as follows:

$$
\phi=\left(\begin{array}{c}
\frac{x_{1,1}}{c_{1}\left(1+x_{1,2}\right)} \\
x_{1,2} \\
x_{2,1}
\end{array}\right)
$$

then system (26) can be putted into the following form:

$$
\left\{\begin{array}{l}
\dot{z}_{1,1}=\frac{1}{c_{1}\left(1+x_{1,2}\right)}\left(\gamma\left(y_{1}, u\right) w_{1}+w_{2}\right) \\
\dot{z}_{1,2}=c_{1} \gamma\left(y_{1}, u\right) z_{1,1} \\
\dot{z}_{2,1}=\beta\left(y_{1}, y_{2}, u\right) z_{1,2}+w_{1}+\beta\left(y_{1}, y_{2}, u\right) w_{2} \\
y_{1}=z_{1,2} \\
y_{2}=z_{2,1}
\end{array}\right.
$$


Then

$$
\eta(y, u, w)=\left[\begin{array}{c}
\eta_{1,1}(y, u, w) \\
\eta_{1,2}(y, u, w) \\
\eta_{2,1}(y, u, w)
\end{array}\right]=\left[\begin{array}{c}
\frac{1}{c_{1}\left(1+y_{1}\right)}\left(\gamma\left(y_{1}, u\right) w_{1}+w_{2}\right) \\
0 \\
w_{1}+\beta\left(y_{1}, y_{2}, u\right) w_{2}
\end{array}\right]
$$

Since

$$
\operatorname{rank} \frac{\partial}{\partial w} \eta_{i, 1}(y, u, w)=\left[\begin{array}{cc}
\frac{1}{c_{1}\left(1+y_{1}\right)} \gamma\left(y_{1}, u\right) & \frac{1}{c_{1}\left(1+y_{1}\right)} \\
1 & \beta\left(y_{1}, y_{2}, u\right)
\end{array}\right], \text { for } 1 \leq i \leq 2
$$

and

$\frac{\partial}{\partial w} \eta_{1,2}(y, u, w)=0$

if $\gamma\left(y_{1}, u\right) \beta\left(y_{1}, y_{2}, u\right) \neq 1$ and $c_{1}\left(1+y_{1}\right) \neq 0$, then Corollary 2 is fulfilled, therefore both the state and the unknown input of system (26) can be recovered by an observer.

Now, let us design an observer for system (27). We assume that $u$ is bounded and $\gamma\left(y_{1}, u\right) \beta\left(y_{1}, y_{2}, u\right) \neq 1$ and $c_{1}\left(1+y_{1}\right) \neq 0$, then the left invertibility problem for (27) may be solved by a step by step sliding mode observer [13] of the following form:

$$
\begin{aligned}
& \dot{\hat{z}}_{1,1}=E_{1} \kappa_{2} \operatorname{sign}\left(\tilde{z}_{1,1}-\hat{z}_{1,1}\right) \\
& \dot{\hat{z}}_{1,2}=c_{1} \gamma\left(y_{1}, u\right) \hat{z}_{1,1}+\kappa_{1} \operatorname{sign}\left(z_{1,2}-\hat{z}_{1,2}\right) \\
& \dot{\hat{z}}_{2,1}=\beta\left(y_{1}, y_{2}, u\right) \hat{z}_{1,2}+\kappa_{3} \operatorname{sign}\left(z_{2,1}-\hat{z}_{2,1}\right)
\end{aligned}
$$

where $z_{1,2}=y_{1}, z_{2,1}=y_{2}$ and

$$
\begin{aligned}
& \tilde{z}_{1,1}=\hat{z}_{1,1}+E_{1} \frac{\kappa_{1} \operatorname{sign}\left(z_{1,2}-\hat{z}_{1,2}\right)}{c_{1} \gamma\left(y_{1}, u\right)} \\
& {\left[\begin{array}{c}
\tilde{w}_{1} \\
\tilde{w}_{2}
\end{array}\right]=\left[\begin{array}{cc}
\frac{1}{c_{1}\left(1+y_{1}\right)} \gamma\left(y_{1}, u\right) & \frac{1}{c_{1}\left(1+y_{1}\right)} \\
1 & \beta\left(y_{1}, y_{2}, u\right)
\end{array}\right]^{-1}\left[\begin{array}{c}
E_{2} \kappa_{2} \operatorname{sign}\left(\tilde{z}_{1,1}-\hat{z}_{1,1}\right) \\
E_{2} \kappa_{3} \operatorname{sign}\left(z_{2,1}-\hat{z}_{2,1}\right)
\end{array}\right]}
\end{aligned}
$$

with

if $z_{1,2}=\hat{z}_{1,2}, E_{1}=1$, else $E_{1}=0$

if $z_{2,1}=\hat{z}_{2,1}$ and $\tilde{z}_{1,1}=\hat{z}_{1,1}$, then $E_{2}=1$, else $E_{2}=0$ 


\section{Conclusion}

This article focused on seeking the MODO normal form. Firstly, the partial differential equations were given to deduce all functions $\alpha_{i, j}$, with which we can design a new frame of vector fields from the natural one associated with a dynamical system with outputs. And then sufficient geometrical condition was given in order to determine whether a system (1) can be transformed into the normal form (2). Finally the obtained result was extended for systems with unknown inputs. Nevertheless, one open problem remained to be solved is to find the necessary condition.

\section{References}

[1] G. Besancon \& G. Bornard, State equivalence based observer design for nonlinear systems, In Proc of IFAC World Congress, San Francisco CA, UAS PP 287-292, 1996.

[2] G. Besancon, G. Bornard \& H. Hammouri, Observer synthesis for a class of nonlinear control systems, Europea Journal of control, vol. 3. no.1, pp. 176-193, 1996.

[3] G. Besancon, A viewpoint on observability and observer design for nonlinear systems, in New directions in nonlinear observer design H. Nijmeijer and T.I.Fosse, Editors- Springer.

[4] K. Busawon, M. Farza \& H. Hammouri, A simple observer for a class of nonlinear systems, Appl. Math. Lett, Vol. 11, No. 3, pp. 27-31, 1998.

[5] D. Boutat G. Zheng \& J.P. Barbot, Observer Error Linearization Multi-Output Depending, In Proc. of IEEE CDC 06, 2006.

[6] A. Glumineau, C. H. Moog \& F. Plestan, New algebro-geometric conditions for the linearization by inputoutput injection, IEEE Transactions on Automatic Control, vol 41, no, 4 pp 598-603, 1996.

[7] M. Guay, Observer linearization by output diffeomorphism and outputdependent time-scale transformations, NOLCOS'01 Saint Petersburg, Russia, pp 1443-1446 2001.

[8] H. Hammouri \& J. De Leon Morales, Observer synthesis for state-affine systems, In Proc. of IEEE CDC 90, 1990.

[9] A. Isidori, Nonlinear control systems, 3rd Edition, Springer,1995.

[10] A. Krener \& A. Isidori, Linearization by output injection and nonlinear observer, Systems \& Control Letters, Vo 3, pp 47-52, 1983. 
[11] A. Krener \& W. Respondek, Nonlinear observer with linearizable error dynamics, SIAM J. Control and Optimization, Vol 30, No 6, pp 197-216, 1985.

[12] H. Nijmeijer \& A.J. Van Der Schaft, Nonlinear Dynamical Control Systems, Springer (1996).

[13] W. Perruquetti \& J-P Barbot, Sliding mode control in engineering, M. Dekker (2002).

[14] F. Plestan \& A. Glumineau, Linearization by generalized input-output injection, Systems

[15] W. Respondek, A. Pogromsky \& H. Nijmeijer, Time scaling for observer design with linearization erro dynamics, IEEE, Transactions on Automatic Control 3, pp. 199-216 2004.

[16] M. Sampei \& K. Furuta, On time scaling for nonlinear systems: application to linearization, IEEE, Transactions on Automatic Control 3, pp. 199-216 1989.

[17] X.H. Xia \& W.B. Gao, Nonlinear observer design by observer error linearization, SIAM J. Control and Optimization, Vol 27, pp 199-216, 1989.

[18] G. Zheng, D. Boutat \& J.P. Barbot, Output Dependent Observability Linear Normal Form, In Proc. of IEEE CDC 05, 2005.

[19] G. Zheng, D. Boutat \& J.P. Barbot, Single Output Dependent Observability Normal Form, SIAM J. Control and Optimization, to appear.

[20] G. Zheng, D. Boutat \& J.P. Barbot, Output Depending Quadratic Observability Normal Form, In Proc. of IFAC NOLCOS 2007. 